\title{
Evaluation of uncertainties on generation expansion planning
}

\begin{abstract}
In the deregulated power market the generator firms compete with each other to reach the maximum profit. And in this type of power market the investors encounter several uncertain parameters. Demand uncertainty is one of the main parameters can reduce the risk of investing when the planners consider in their planning. Stochastic programming has been represented in this article to solve the investment problem. This article proposed a model by considering the demand uncertainties. And also, other parameters including the financial restriction, forced outage rate (FOR), penalty for unserved demand has been considered. The variable decisions in this model include the total benefit of investor, expansion capacity at the beginning of each period, operation level for each generator. Sensitivity analysis for FOR and the penalty for unserved demand on the expansion planning confirm this model. Finally, the proposed framework has been implemented on the case study to approve this model.
\end{abstract}

Keyword: Generation expansion planning; Demand uncertainties; Stochastic programming 